\title{
State-of-the-Art Analysis of High-Frequency (Gamma Range) Electroencephalography in Humans
}

\author{
Judith F. Nottage ${ }^{a} \quad$ Jamie Horder ${ }^{b}$ \\ Departments of a Neuroimaging and ${ }^{b}$ Forensic and Neurodevelopmental Sciences, Institute of Psychiatry, Psychology and \\ Neuroscience, King's College London, London, UK
}

\section{Key Words}

Gamma oscillations - Artefacts - Muscle · Saccades · Blinks ·

Electromyography

\begin{abstract}
Gamma oscillations $(>30 \mathrm{~Hz})$ in the brain are involved in attention, perception and memory. They are altered in various pathological states, as well as by neuropharmaceuticals, so that they are of interest in drug and clinical investigations. However, when the human electroencephalogram is recorded on the scalp, this neural high-frequency signal is buried under a range of other electrical signals such that, without careful handling, recordings of the high-frequency electroencephalogram cannot be considered reliable. The artefacts of concern originate from: power line noise, saccade-associated contraction of the extra-ocular muscles, activity of muscles in the scalp, face and neck, screen refresh artefacts and activity of the muscles associated with blinking. Recent progress in dealing with these artefacts is described, including either noise cancellation or phased noise template subtraction for power line noise, regression or independent component analysis for correcting extra-ocular muscle activity and mathematical modelling for reducing scalp, face and neck muscle activity. If the artefacts are properly addressed, the neural gamma signal can be uncovered.
\end{abstract}

(c) 2016 S. Karger AG, Basel
(C) 2016 S. Karger AG, Basel

0302-282X/16/0724-0219\$39.50/0

\section{Introduction: The Importance of the High} Frequencies of the Electroencephalogram

The key role which oscillations in the gamma band $(>30 \mathrm{~Hz})$ play in brain processes was initially demonstrated in animals [1-3], and these findings were subsequently extended to humans. The gamma band is selectively increased when active processing of stimuli occurs with attention $[4,5]$. Gamma synchrony increases the gain of neural signals, and artificially inducing gamma oscillations using optically activated ion channels can increase the perception of a stimulus [6]. Human intracortical investigations have confirmed that gamma oscillations are involved in cognitive processes in humans, with different gamma subbands playing different roles $[7,8]$.

Gamma oscillations can be affected by various pharmacological agents, in vitro and in vivo [9-11]. For example, stimulant drugs can shift the frequency of gamma oscillations in frontostriatal circuits [12]. Furthermore, changes in the gamma band have been associated with a number of pathological conditions. Firstly, oscillations in motor circuits in the beta and gamma bands have been implicated in the pathology of Parkinson's disease [13]. Treatment by either dopamine agonists or by deep brain stimulation at high frequencies has been hypothesized to suppress symptoms via an effect on these oscillations

\section{KARGER 125}

E-Mail karger@karger.com www.karger.com/nps 
[14]. Secondly, there is an extensive literature concerning gamma band changes in schizophrenia $[15,16]$, although the possibility of artefactual contamination casts a shadow over much of this work. Furthermore, ketamine, which in subanaesthetic doses induces psychotic symptoms in humans, has been shown to increase the amplitude of gamma signals in humans and animals $[10,17]$. Thirdly, it has recently been found that localized bursts of high-frequency activity precede seizures in epileptic patients $[18,19]$. Fourthly, the conscious perception of pain has been shown to be uniquely related to gamma band oscillations [20].

Unfortunately, the contamination of the gamma band of the human scalp electroencephalogram by non-neural signals is a major and long-standing concern, and past limitations in computing power have hindered the development of satisfactory methods for resolving the problem. Electroencephalography (EEG) research groups have therefore had two options; either to ignore frequencies above $30 \mathrm{~Hz}$ or else to reduce the extent of the problem by attempting to minimize the artefacts at the acquisition stage, rejecting gross artefacts and then taking an optimistic viewpoint about the level of residual contamination. However, since recent research has highlighted the full extent of the artefactual contamination in the gamma band, this optimistic stance is no longer tenable $[21,22]$.

This article discusses the artefacts concerned as well as recent progress in harnessing modern computing power to reduce them.

\section{High-Frequency Artefacts in the Human Scalp Electroencephalogram}

\section{Power Line Noise}

All mains-powered electrical equipment generates alternating electromagnetic fields, at 50 or $60 \mathrm{~Hz}$ depending on the country, and these are often much greater in amplitude than neural oscillations at the surface of the scalp. Several steps are taken to minimize the power line noise at acquisition, such as the incorporation of 'common mode rejection' in EEG amplifiers as well as endeavouring to keep impedances low and balanced across electrodes. Some research groups carry out EEG recording in electrically shielded rooms whilst others employ 'active' electrodes which have an amplifier incorporated into each electrode. When power line noise is present in an EEG recording, standard practice has been to apply a low pass filter of around $30 \mathrm{~Hz}$, or else a band stop, or 'notch'

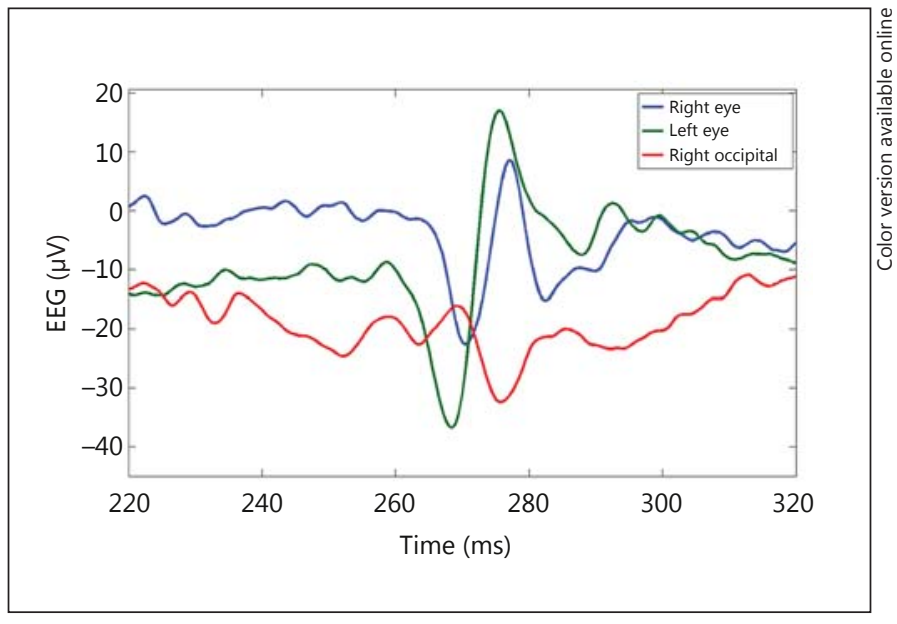

Fig. 1. An example of an artefact produced by the contraction of the extra-ocular muscles in a microsaccade. The blue and green lines (colours in the online version only) represent recordings at the outer canthus of the right and left eyes, respectively. The difference between left and right from 270 to $290 \mathrm{~ms}$ is due to the superposition of a corneal retinal signal from the eyeball turning. The red line shows an opposite polarity signal recorded at the right occipital location (PO4) which is due to the muscle artefact in the ear lobe reference. A face stimulus is presented at $t=0 \mathrm{~ms}$. See Nottage [33] for details.

filter to the data offline before further analysis. Whilst this is useful when calculating low-frequency event-related potentials, it is inappropriate for the analysis of the gamma band. This is not only because some frequency bands are lost, but also because such filters can cause spurious oscillations in the frequency bands adjacent to the 50- or $60-\mathrm{Hz}$ power line noise (termed 'ringing') and distort the phase of neural oscillations in those bands. Nevertheless, in an EEG recording with low to moderate power line noise, it might appear to be possible to quantify the gamma signal at frequencies well away from the power line noise. However, this is not the case in practice, since the power line noise is superimposed on other broad-band myogenic artefacts, and the former must be removed, without distorting the underlying signal, before the latter can be addressed.

\section{Activity of the Extra-Ocular Muscles Associated with Saccades}

Amongst clinical neurophysiologists it has been known for some time that the 'lateral rectus' muscles, a pair of extra-ocular muscles which contract to move the eyeball during a saccade, can produce a large spike in the EEG, or 'spike potential' [23]. Also there is known to be 


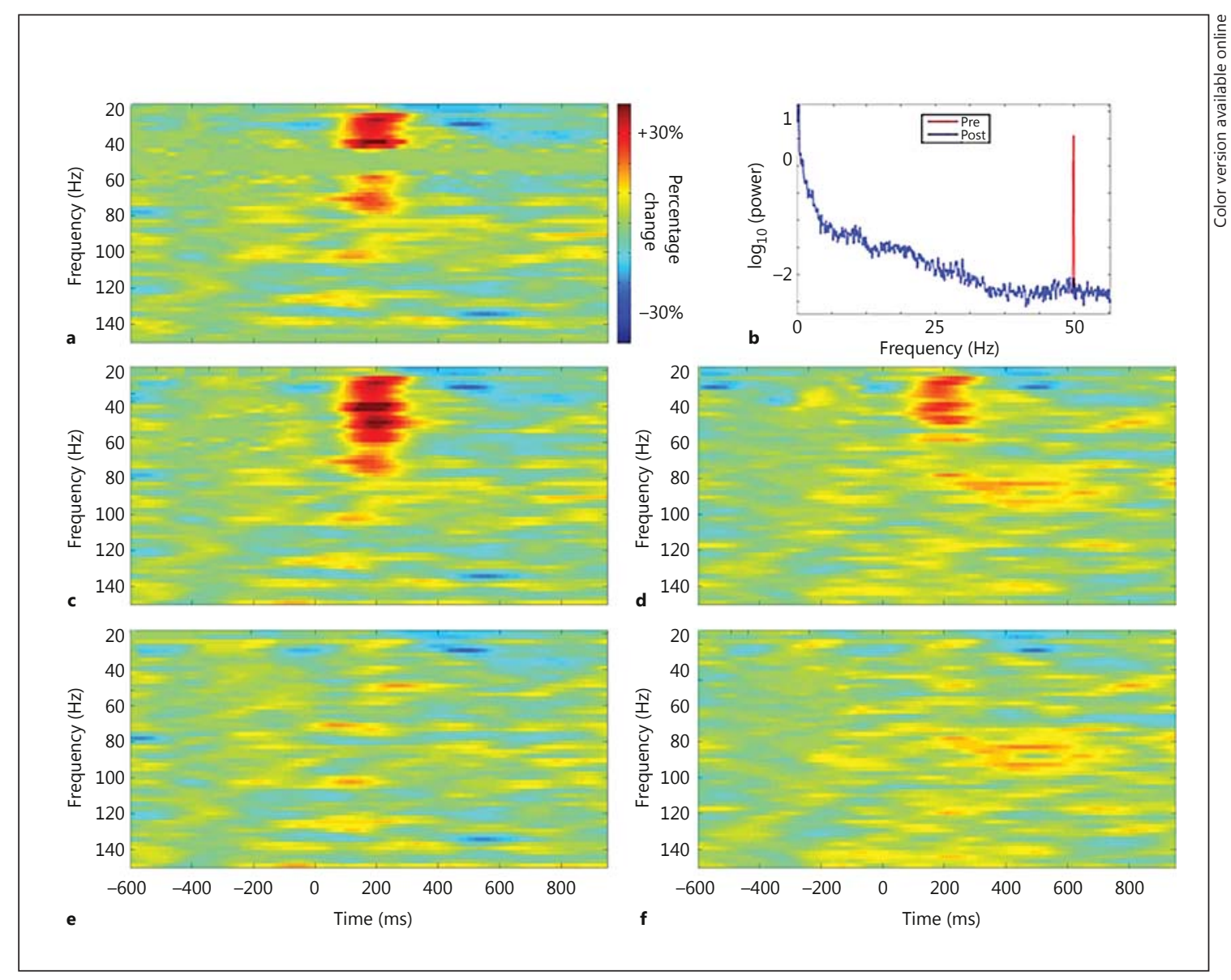

Fig. 2. Examples of correction of the EEG signal. a Percentage change in gamma magnitude in a visual task before any correction. A band around $50 \mathrm{~Hz}$ is lost due to power line noise (face stimuli appear at zero time, $\mathrm{PZ}$ mastoids, baseline is -500 to $-200 \mathrm{~ms}$ ). b Logarithm of Fourier-transformed EEG signal, before and after the reduction of power line noise using a phased noise template subtraction algorithm ('PHANTEM'). Sampling frequency $=5$ $\mathrm{kHz}$, fast Fourier transform length $=13.10$ s. c-f As a, but with power line correction using noise cancellation. The saccadic muscle potential (SMP) artefact is corrected in e and $\mathbf{f}$, removing the sharp gamma peak at $200 \mathrm{~ms}$. Scalp EMG is corrected in $\mathbf{d}$ and $\mathbf{f}$, revealing a neural $75-$ to $95-\mathrm{Hz}$ signal at $200-700 \mathrm{~ms}$. a suppression of saccades immediately after the appearance of a visual stimulus, followed by a rebound 200-300 $\mathrm{ms}$ after stimulus presentation. Despite this, it came as a shock to the EEG community when Yval-Greenberg et al. [22] demonstrated, in 2008, that the much reported broad-band increase in induced gamma power, at around 200-300 ms after a visual stimulus, originated predominately from the sharp contraction of extra-ocular muscles during microsaccades and not from neuronal activity.

Examples of this artefact can be seen in figures 1 and 2. The extra-ocular muscles are set back inside the skull, with only a very thin layer of bone separating them from the brain, and therefore these muscle potentials can even affect intracranial recordings in the anterior temporal lobe and inferior prefrontal cortex [24]. They also contaminate the nose, ear lobe and mastoid electrodes which are commonly used in scalp EEG recordings, and can affect the scalp EEG signal at varying magnitudes at any electrode locations other than those close to $\mathrm{CPz}$ and $\mathrm{Pz}$. Magneto-encephalographic recordings can also be contaminated by this artefact [for details, see 25].

\section{Scalp, Neck and Face Muscle Activity}

Whilst it has long been understood that the high frequencies of the electroencephalogram are strongly contaminated by the activity of scalp, face and neck muscles [26], the full extent of this contamination was only ex- 
posed when Whitham et al. [21] paralysed the superficial muscles and discovered that gamma power was reduced by as much as $98 \%$ at some electrode locations. These muscles are very sensitive to emotional state and stress levels, and cognitive tasks can increase the contamination of EEG by electromyography (EMG) [27, 28]. If there are a few large EMG bursts, then the contaminated segments can simply be excluded from the analysis. However, in addition to occasional large contractions, the scalp, neck and face muscles often undergo prolonged contraction, giving rise to tonic EMG throughout an EEG recording. It is possible to train subjects to relax their scalp and neck muscles, which may be an appropriate approach for understanding gamma signals in healthy subjects. However, relaxation training has the disadvantage of introducing an additional cognitive task. Also, in pharmaco-EEG or psychiatric investigations, the administration of drugs or the presence of a psychiatric disorder may impair a subject's ability to continuously relax their scalp. In this case, differential compliance with the instruction to relax could seriously confound the interpretation of the results of the study.

\section{Screen Refresh Artefact}

The older cathode ray tube type of screen is often preferred for event-related potentials, because the time of the screen refresh can be more sharply defined. However, an electromagnetic signal is produced by the screen refresh, and this can sometimes produce a signal in the gamma band of the electroencephalogram. When this artefact occurs, it is most prominent at anterior electrode locations and its spectra consist of a narrow band at the primary refresh frequency plus higher-frequency harmonics.

\section{Orbicularis Oculi: Blink-Associated Muscle Activity}

Whilst the effect of contamination by extra-ocular activity has recently received much attention, the effect of muscles associated with the closing of the eye during blinking has, to date, been ignored. There are two main muscles, the levator palpebrae superioris (LPS) and the orbicularis oculi (OO). The LPS muscles, which pull the eyelids upwards, are tonically active, but at the start of a blink the LPS muscles relax, and this is followed by a rapid contraction of the OO sphincter muscles over the eye $[29,30]$. Blinks tend to occur preferentially at certain times in cognitive tasks so that a spurious frontal eventrelated gamma band change could potentially be generated. Also, blink startle reflexes have an EMG component [31], which can be modulated pharmacologically [32] as well as by other factors such as emotional state. Fortunately, these muscles are located much more superficially than the extra-ocular ones so that they only contaminate frontal areas.

\section{Extracting Neural High-Frequency Oscillations from Human Scalp EEG}

\section{Power Line Noise}

When independent component analysis (ICA) is used to deal with lower-frequency artefacts, one component often consists of the power line noise. Removing this component may, in some circumstances, be an effective way to reduce power line noise contamination, provided that two conditions are met. Firstly the signal must have the same phase across electrodes, and secondly the power line component must not contain other signals as well as the power line noise, or removal of this component will alter the underlying electroencephalogram. In practice these conditions may not be met, and therefore noise cancellation has recently been proposed as an alternative method for addressing the power line noise artefact [33, 34]. One or more electrodes are used to record the power line noise simultaneously with the electroencephalogram, and this signal is transformed so as to replicate the power line signal at each electrode. However, care is needed to avoid overloading the EEG amplifier with the noise signal, and therefore this method is unsuitable for simultaneous functional magnetic resonance imaging/EEG which generates very large amplitude gradient artefacts. The power line signal is band pass filtered to obtain a pure $50-\mathrm{Hz}$ sine wave and is combined with a calculated weight of a 90-degree phase-shifted sine wave, which is a negative polarity cosine wave. Preferably the sampling rate should be a multiple of 200 for $50-\mathrm{Hz}$ noise or 240 for 60 $\mathrm{Hz}$ noise so that the 90 -degree phase shift required (5 ms at $50 \mathrm{~Hz}$ or $41 / 6 \mathrm{~ms}$ at $60 \mathrm{~Hz}$ ) will consist of a whole number of sample points. Any phase of power line noise can be matched using the trigonometric identity in equation 1 , as demonstrated in equation 2 below:

$$
\sin (\mathrm{A}+\mathrm{B})=\sin (\mathrm{A}) \cos (\mathrm{B})+\cos (\mathrm{A}) \sin (\mathrm{B}) .
$$

Replacing A with $\omega \mathrm{t}$ (where, for $50 \mathrm{~Hz}, \omega=2 \pi / 20$ and $t$ is the time in milliseconds) and B with the phase shift $\alpha$ of the power line noise in a particular electrode gives:

$$
\sin (\omega t+\alpha)=C \sin (\omega t)+D \cos (\omega t),
$$

where $\mathrm{C}$ and $\mathrm{D}$ are constants, equal to $\cos (\alpha)$ and $\sin (\alpha)$, respectively. 
The constants $\mathrm{C}$ and $\mathrm{D}$ are calculated by simultaneous regression of contaminated EEG with the $50-\mathrm{Hz}$ sine and cosine waves. Equation 1 can also be used to reconstruct the higher-frequency harmonic components. The power line noise is then subtracted, leaving behind uncontaminated EEG, as seen in figure $2 a$ and c.

Noise cancellation is the most effective method we have used, but we have sometimes needed to analyse EEG where a recording of the power line noise is not available. This led us to develop an alternative approach, using average template subtraction. For each electrode, we cut the $5-\mathrm{kHz}$ sampled electroencephalogram into 20 -ms intervals (100 time points). By averaging these segments, we can arrive at an accurate template of the $50-\mathrm{Hz}$ power line noise waveform. However, since the phase of the noise is non-stationary and is observed to 'drift' over time, we also identified the phase time series via an adaptive search algorithm. We then constructed a noise time series with the appropriate phase evolution and subtracted this from the signal. As can be seen in figure $2 \mathrm{~b}$, power line $(50 \mathrm{~Hz}$ power) reduction can be obtained with negligible disruption of the underlying signal, provided that EMG contamination is not too high.

\section{Activity of the Extra-Ocular Muscles Associated with Saccades}

The contraction of the extra-ocular muscles at the start of a saccade or microsaccade produces a biphasic signal with a frequency spectrum of 30-100 Hz, which we have termed the saccadic muscle potential (SMP). There are 3 published methods for dealing with SMPs: an ICA method [35], a regression method [33], as used in figure 2, and a second, more complex ICA method [36].

All 3 methods use dedicated electrodes at the outer canthi of the left and right eyes (called HEOGL and HEOGR, respectively), but various other eye electrodes are also included by the different groups. It is essential that, during recording, the HEOGL and HEOGR channels are referenced to the common reference for all electrodes and not to each other, since recording HEOGL and HEOGR as a bipolar channel causes the SMP artefact to be subtracted out. The methods proposed by Keren et al. [35] and Hassler et al. [36] used $\mathrm{Pz}$ as the reference for detection of the SMPs, whilst Nottage [33] used the mastoid or ear lobe recording reference. Both Nottage and Hassler et al. used arithmetic manipulations of the mean eye channel signal to make the SMP artefacts more pronounced.

With ICA a surrogate SMP channel can be used to determine the unmixing matrix [36], or the weights can be found using regression. These weights can be calculated either using the average of all the SMPs or else using individual SMP values and employing an iterative approach to find the weights which minimize the sum of the squares of the errors. The former has the advantage that it is robust to the presence of noise but the disadvantage that saccades in different directions will be averaged together, but SMPs for saccades in different directions are not identical. For example, Collewijn [37] studied the timing of saccades in the left and right eyes and reported that 'the nasalward moving eye lagged the temporalward moving eye consistently by slightly less than $1 \mathrm{~ms}$, and differences in the magnitudes of SMPs associated with saccades in different directions have also been noted [38]. The final step of the correction is that the weighted SMP signal is subtracted from the electroencephalogram at each electrode location.

One unresolved issue is whether it is preferable to subtract a signal containing only the identified SMP artefacts, but which is set to zero at other time points, or the continuous signal, or component, from the eye region, as would be the case with ICA. The extent to which extraocular muscles generate signals in between SMPs is unclear, but we have occasionally observed runs of gamma frequency waves, lasting 100-200 ms, which appear to come from the extra-ocular muscles. Subtraction of the continuous signal will remove all such extra-ocular activity, but, since ICA unmixing is unlikely to be perfect because of the large number of muscle sources, it will probably also introduce extra noise from more of the superficial anterior muscles. Whichever approach was used, both Nottage [33] and Hassler et al. [36] reported that the time frequency plot of the residual visual gamma signal had a more sustained, narrow band appearance than that of the SMPs, presumably reflecting neural gamma signals.

\section{Scalp, Neck and Face Muscle Activity}

Where muscle artefact originates from large amplitude activity which is conducted to a widely distributed set of electrodes, various types of blind source separation [39] or ICA in the time domain can be used for correction. Examples are the movement of the tongue during speech and the activity of the masseter muscle during jaw clenching. However, the major EMG contamination of the electroencephalogram originates in scalp, neck and face muscles which lie just under individual electrodes [40]. Each muscle fibre generates a signal, and therefore the EMG artefact detected at each electrode is unique. ICA has been used to rectify the effect of the electromyogram on low EEG frequencies, although not without controversy $[41,42]$. The 


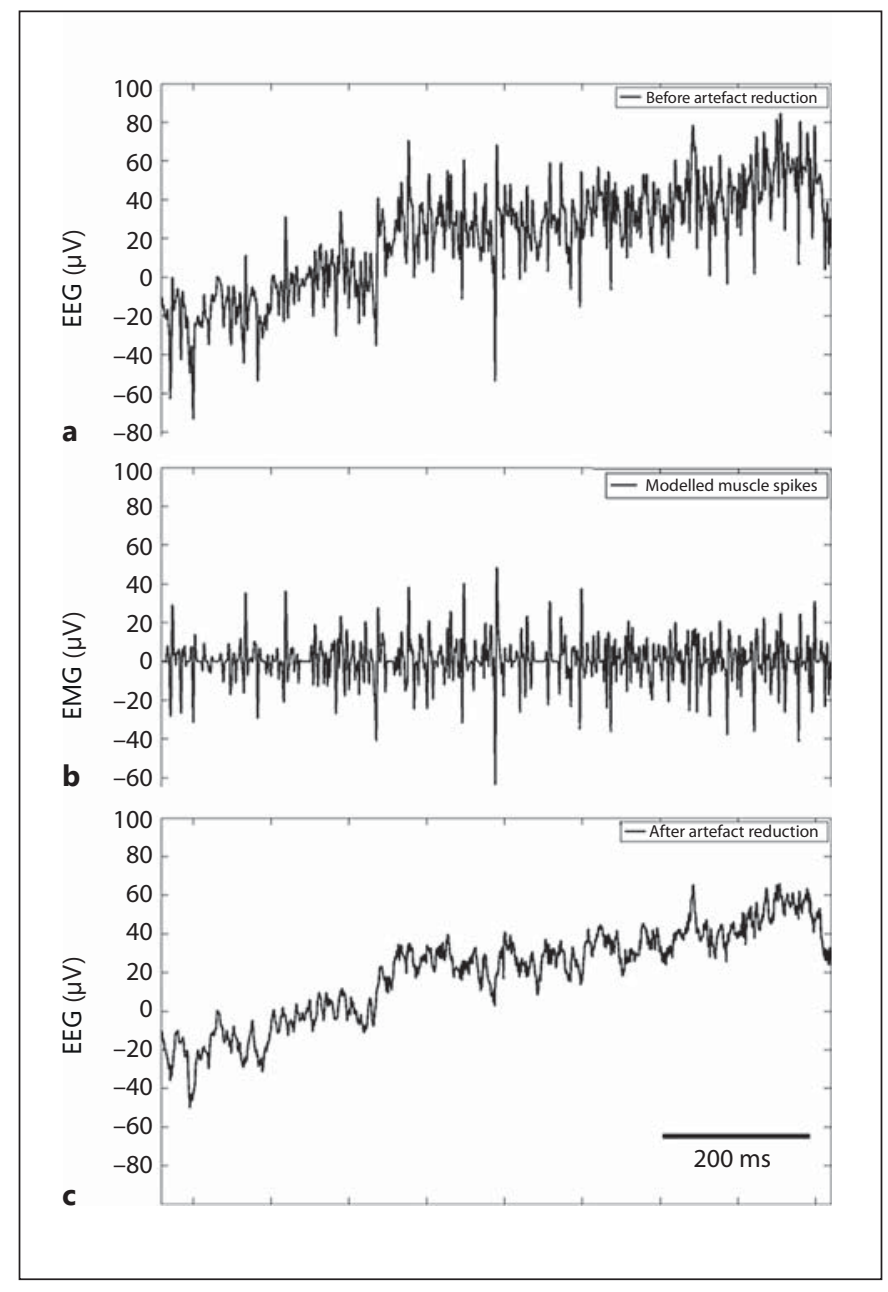

Fig. 3. An example of EEG before and after the reduction of tonic EMG using mathematical modelling with Gabor functions: uncorrected EEG (a), the modelled EMG signal (b) and corrected EEG (c).

most effective ICA approach is to use a high gamma magnitude as a regressor in the frequency domain, but this cannot be used to clean the gamma band of the electroencephalogram. Alternatively, beamforming has been used if the source of a gamma signal is known [43], but this is not suited to quantifying the widely distributed resting gamma signal. Recently, attempts have been made to use ICA in the time domain to clean the gamma signal of scalp muscle activity. Using high-density EEG recording (126 channels), the expected temporal pattern of gamma activation was retrieved at dorsal occipital electrodes in a visual motion task [44]. This required the rejection of $46 \pm$ 7.8 ICA components and reduced the gamma amplitude by up to $75 \%$. However, if the unmixing of signals with
ICA is imperfect, some of the neural signal will be rejected with the noise. The extent of this loss of signal is unknown, but it could seriously confound pharmaco-EEG or psychiatric investigations, particularly where the signal of interest is located in more severely contaminated areas, such as the temporal or frontal cortex. If extra electrodes on the face and neck were to be added, this could improve the ICA unmixing, although additional muscle sources under these extra electrodes are likely to limit the usefulness of this strategy.

An alternative approach is to fit a mathematical model to individual muscle spikes and subtract these from the signal [34]. The muscle spikes are modelled using a Gabor function, which consists of a sine wave multiplied by a Gaussian function. This approach has the advantage that it does not require the subjective rejection of components, thus not reducing the desired signal to an indeterminable extent. Examples of this process can be seen in figures 2-4. It is important that any method for reducing the EMG artefact is able to do so whilst preserving the neural gamma signal. The mathematical modelling method was able to effectively correct the gamma signal associated with a self-paced motor task [34], as can be seen in figure 5 . The baseline gamma signal is substantially reduced, revealing a significant $65-$ to $85-\mathrm{Hz}$ gamma activation over left motor/somatosensory areas. However, in some subjects the scalp EMG contamination is so severe that it cannot be effectively removed by this mathematical modelling process. In such cases it may still be possible to estimate the neural gamma signal, given sufficient data, by assuming that over time, the electromyogram remaining after correction is proportional to the original one. The amplitude of the corrected electroencepalogram at each epoch is regressed with the amplitude of the EMG signal which has been removed. Then the corrected EEG amplitude at zero EMG correction, corresponding to zero EMG contamination, can be taken as the intercept.

\section{Screen Refresh Artefact}

This artefact occasionally occurs with a large enough amplitude to confound neural gamma signals (fig. 6a), and in these cases we have found that using a template subtraction procedure is effective. The screen refresh artefact is captured by recording a sample of EEG with the subject's head located within a few centimetres of the screen, and using the maximum sampling rate (e.g. $10 \mathrm{kHz}$ ). After the power line contamination has been removed from this signal, high pass filtering enables the waveform of the electrical potential caused by the screen refresh to be clearly seen in an average of the most anterior EEG channels. Averaging 




Fig. 4. As figure 3, but expanded on two different time scales to show the fine detail of EMG subtraction.

Fig. 5. Effect of EMG reduction on a motor gamma signal $(65-85 \mathrm{~Hz})$ in the average of 8 subjects. a Topographic distribution of $\mathrm{Z}$ scores for the gamma event-related synchronization in $250 \mathrm{~ms}$ after a self-paced button press with the right hand $(\mathrm{n}=16)$. The maximum $\mathrm{Z}$ score is at C3-Cz. b High gamma amplitude. Modulation of 65- to $85-\mathrm{Hz}$ signal with time with the button press being at $0 \mathrm{~ms}$. After EMG reduction, there is a statistically significant peak, as expected, in the $250 \mathrm{~ms}$ after the button press, as well as a large reduction in the baseline. Before EMG reduction, this gamma peak was not significant. See Nottage et al. [34] for further details.

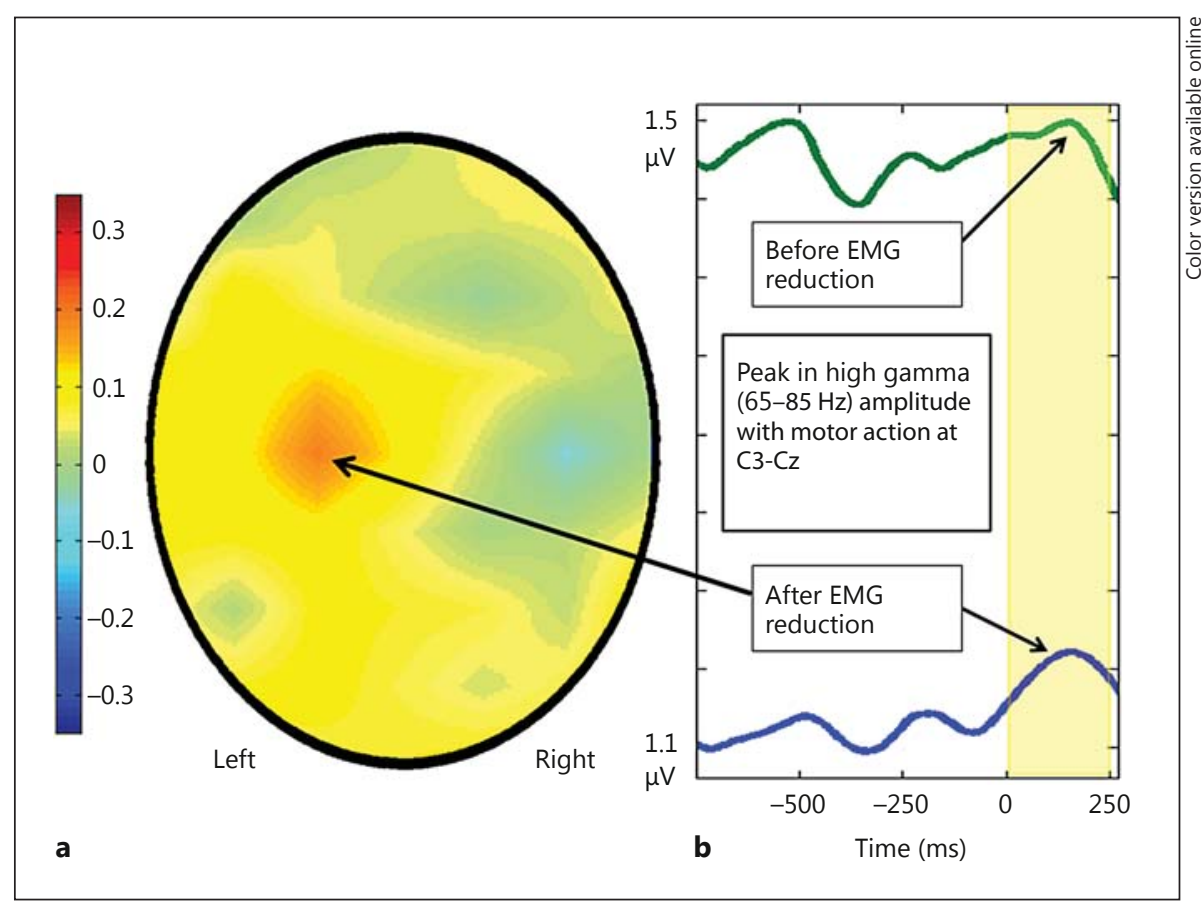

Analysis of High-Frequency EEG in Humans
Neuropsychobiology 2015;72:219-228 DOI: $10.1159 / 000382023$ 


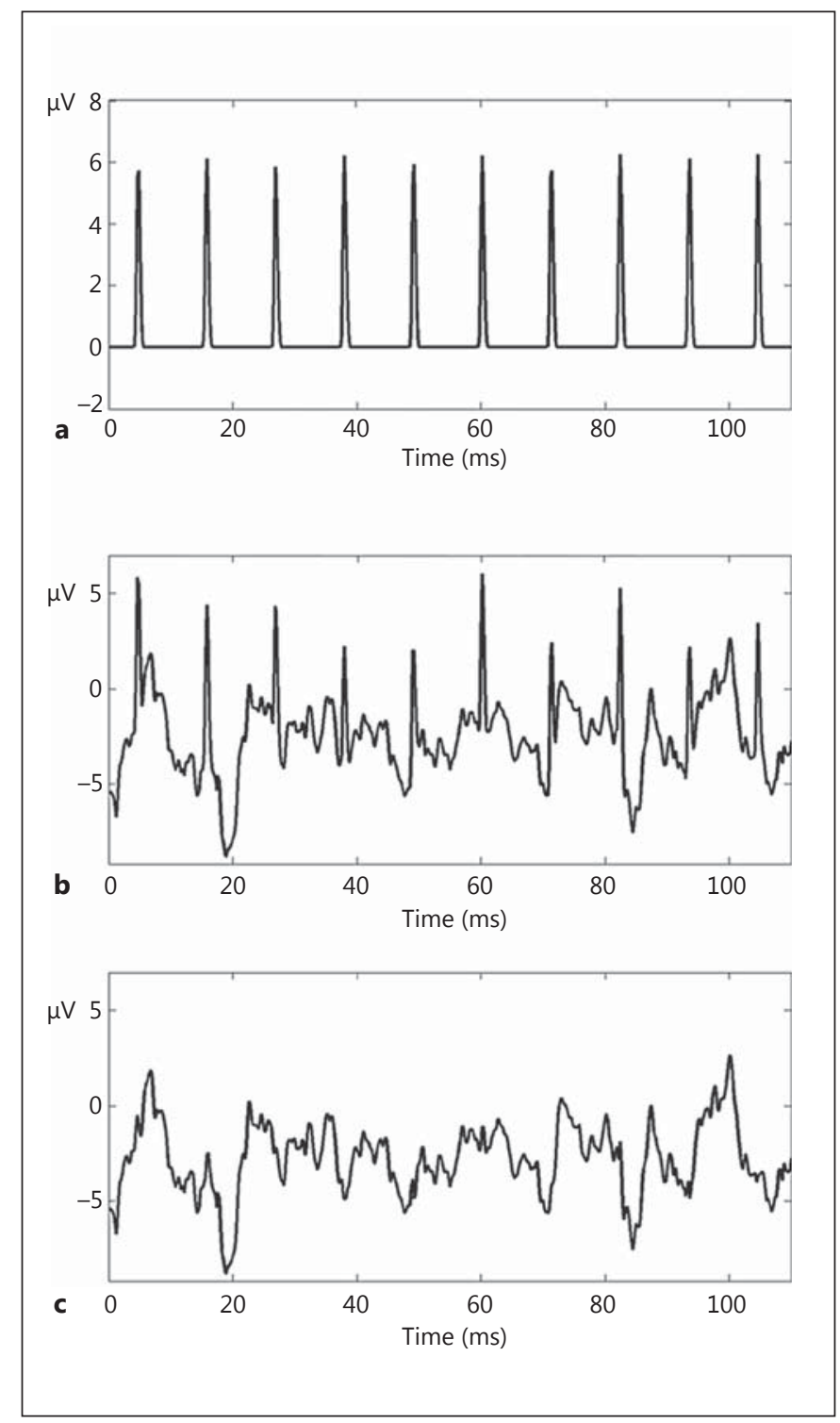

Fig. 6. a Cathode ray tube screen refresh signal. The reconstructed refresh artefact, which is subtracted from the EEG. b Contaminated EEG. EEG recording at the corner of the left eye (HEOGL) contaminated with screen refresh artefact from a cathode ray tube screen with a $90-\mathrm{Hz}$ refresh rate. EEG was recorded with an ear lobe reference, $5-\mathrm{kHz}$ sample rate and $1-\mathrm{kHz}$ low pass filter. c Corrected EEG. As b, but corrected for screen refresh artefact.

over individual spikes gives a clean example of the screen refresh waveform. This signal can be interpolated (e.g. by a factor of 10) and then shifted in time until it matches the screen refresh artefact in the mean anterior channels in the recording to be cleaned (fig. 6b). Subtraction of a scaled version of this waveform produces effective cleaning of the
EEG signal with minimal distortion, as can be seen in figure $6 c$. However, as with all artefacts, the best solution is prevention, and it is therefore advisable to switch off screens when they are not required, such as when recording resting state EEG after visual tasks.

\section{Blink-Associated Muscle Activity}

As yet there are no published methods for addressing the blink-associated muscle activity, so caution is advised when reporting event-related changes from the most anterior channels. If blinking occurs preferentially at a particular time in a cognitive task, or in response to a startling or painful stimulus, $\mathrm{OO}$ activity could lead to an artefactual frontal event-related synchronization whilst LPS muscle relaxation at the start of a blink would lead to a spurious event-related desynchronization. Contaminated channels should not be used in an average reference, and steps should be taken to avoid confusing $\mathrm{OO}$ activity with SMPs during the correction of this component.

\section{Different Neural Gamma Band Components of the Electroencephalogram}

If all the above procedures are effectively applied, producing a signal containing almost only high-frequency activity generated by the brain, there is still one further complication to be considered. Whilst neural oscillations at gamma frequencies appear to be important, the magnitude of a Fourier or wavelet transform can be increased in the gamma band without any actual oscillations occurring at this frequency. On the one hand, random neural noise, which occurs in the electroencephalogram with a classic $1 / \mathrm{f}$ spectrum, will contribute to the amplitude. Also, alpha and beta oscillations often occur as sharp sawtooth waveforms, rather than pure sine waves, and these will produce harmonic signals in the gamma band [32]. Use of short time windows for the Fourier transform, of around 128 or $256 \mathrm{~ms}$, can increase the relative contribution of gamma waves compared to alpha and beta waves, since gamma waves can be assumed to be stationary over shorter segments of time than alpha and beta waves.

\section{Conclusion}

The development of methods to clean the higher frequencies of the EEG is gaining momentum. Improvements have been made in dealing with power line noise, saccade-associated contraction of the extra-ocular mus- 
cles, scalp, face and neck EMG and screen refresh artefacts whilst methods to address the artefacts caused by muscle activity associated with blinking are being developed. However, more work is needed to evaluate these methods and determine the optimum analysis pipeline. Also, methods are required to distinguish gamma oscillations from harmonics of alpha and beta waves and the gamma band component of broad-band neural noise. Despite the many hurdles, the gamma band of the human scalp electroencephalogram is now becoming increasingly accessible.

\section{Acknowledgements}

Judith Nottage receives salary support from the National Institute for Health Research (Mental Health Biomedical Research Centre and/or Dementia Biomedical Research Unit) at South London and Maudsley NHS Foundation Trust and King's College London.

\section{References}

1 Freeman WJ: Spatial properties of an EEG event in the olfactory bulb and cortex. Electroencephalogr Clin Neurophysiol 1978;44: 586-605.

12 Gray CM: The temporal correlation hypothesis of visual feature integration: still alive and well. Neuron 1999;24:31-47, 111-125.

$\checkmark 3$ Fries P, Reynolds JH, Rorie AE, Desimone R: Modulation of oscillatory neuronal synchronization by selective visual attention. Science 2001;291:1560-1563.

4 Brovelli A, Lachaux JP, Kahane P, Boussaoud D: High gamma frequency oscillatory activity dissociates attention from intention in the human premotor cortex. Neuroimage 2005;28: 154-164.

5 Kahlbrock N, Butz M, May ES, Schnitzler A: Sustained gamma band synchronization in early visual areas reflects the level of selective attention. Neuroimage 2012;59:673-681.

6 Knoblich U, Siegle JH, Pritchett DL, Moore CI: What do we gain from gamma? Local dynamic gain modulation drives enhanced efficacy and efficiency of signal transmission. Front Hum Neurosci 2010;4:1-12.

$>7$ Jerbi K, Ossandón T, Hamamé CM, Senova S, Dalal SS, Jung J, Minotti L, Bertrand O, Berthoz A, Kahane P, Lachaux JP: Task-related gamma-band dynamics from an intracerebral perspective: review and implications for surface EEG and MEG. Hum Brain Mapp 2009; 30:1758-1771.

8 Gaona CM, Sharma M, Freudenburg ZV, Breshears JD, Bundy DT, Roland J, Barbour DL, Schalk G, Leuthardt EC: Nonuniform high-gamma $(60-500 \mathrm{~Hz})$ power changes dissociate cognitive task and anatomy in human cortex. J Neurosci 2011;31:2091-2100.

$\checkmark 9$ Hajos M, Hoffmann WE, Kocsis B: Activation of cannabinoid-1 receptors disrupts sensory gating and neuronal oscillation: relevance to schizophrenia. Biol Psychiatry 2008;63:10751083.

10 Hunt MJ, Falinska M, Łeski S, Wójcik DK, Kasicki S: Differential effects produced by ketamine on oscillatory activity recorded in the rat hippocampus, dorsal striatum and nu- cleus accumbens. J Psychopharmacol 2011; 25:808-821.

11 Nottage JF, Stone J, et al: Delta-9-tetrahydrocannabinol, neural oscillations above $20 \mathrm{~Hz}$ and induced acute psychosis. Psychopharmacology 2015;232:519-528.

12 Berke JD: Fast oscillations in cortical-striatal networks switch frequency following rewarding events and stimulant drugs. Eur J Neurosci 2009;30:848-859.

13 Brown P: Oscillatory nature of human basal ganglia activity: relationship to the pathophysiology of Parkinson's disease. Mov Disord 2003;18:357-363.

14 Dejean C, Hyland B, Arbuthnott G: Cortical effects of subthalamic stimulation correlate with behavioral recovery from dopamine antagonist induced akinesia. Cereb Cortex 2009; 19:1055-1063.

15 Itil TM: Qualitative and quantitative EEG findings in schizophrenia. Schizophr Bull 1977;3:61-79.

16 Uhlhaas PJ, Haenschel C, Nikolić D, Singer $\mathrm{W}$ : The role of oscillations and synchrony in cortical networks and their putative relevance for the pathophysiology of schizophrenia. Schizophr Bull 2008;34:927-943.

17 Hong LE, Summerfelt A, Buchanan RW, O'Donnell P, Thaker GK, Weiler MA, Lahti AC: Gamma and delta neural oscillations and association with clinical symptoms under subanesthetic ketamine. Neuropsychopharmacology 2010;35:632-640.

18 Modur PN, Zhang S, Vitaz TW: Ictal highfrequency oscillations in neocortical epilepsy: implications for seizure localization and surgical resection. Epilepsia 2011;52:17921801

19 Zijlmans M, Jiruska P, Zelmann R, Leijten FS, Jefferys JG, Gotman J: High-frequency oscillations as a new biomarker in epilepsy. Ann Neurol 2012;71:169-178.

$>20$ Zhang ZG, Hu L, Hung YS, Mouraux A, Iannetti GD: Gamma-band oscillations in the primary somatosensory cortex - a direct and obligatory correlate of subjective pain intensity. J Neurosci 2012;32:7429-7438.
21 Whitham EM, Pope KJ, Fitzgibbon SP, Lewis T, Clark CR, Loveless S, Broberg M, Wallace A, DeLosAngeles D, Lillie P, Hardy A, Fronsko R, Pulbrook A, Willoughby JO: Scalp electrical recording during paralysis: quantitative evidence that EEG frequencies above $20 \mathrm{~Hz}$ are contaminated by EMG. Clin Neurophysiol 2007;118:1877-1888.

22 Yuval-Greenberg S, Tomer O, Keren AS, Nelken I, Deouell LY: Transient induced gamma-band response in EEG as a manifestation of miniature saccades. Neuron 2008;58: 429-441

23 Thickbroom GW, Mastaglia FL: Presaccadic spike potential - investigation of topography and source. Brain Res 1985;339:271-280.

24 Jerbi K, Freyermuth S, Dalal S, Kahane P, Bertrand O, Berthoz A, Lachaux JP: Saccade related gamma-band activity in intracerebral EEG: dissociating neural from ocular muscle activity. Brain Topogr 2009;22:18-23.

25 Carl C, Açık A, König P, Engel AK, Hipp JF: The saccadic spike artifact in MEG. Neuroimage 2012;59:1657-1667.

26 Goncharova II, McFarland DJ, Vaughan TM, Wolpaw JR: EMG contamination of EEG: spectral and topographical characteristics. Clin Neurophysiol 2003;114:1580-1593.

27 Cohen BH, Davidson RJ, Senulis JA, Saron CD, Weisman DR: Muscle tension patterns during auditory attention. Biol Psychol 1992; 33:133-156.

28 Whitham EM, Lewis T, Pope KJ, Fitzgibbon SP, Clark CR, Loveless S, DeLosAngeles D, Wallace AK, Broberg M, Willoughby JO: Thinking activates EMG in scalp electrical recordings. Clin Neurophysiol 2008;119:11661175

29 Porter JD, Burns LA, May PJ: Morphological substrate for eyelid movements: innervation and structure of primate levator palpebrae superioris and orbicularis oculi muscles. J Comp Neurol 1989;287:64-81.

30 Bour L, Ongerboer de Visser B, Aramideh M, Speelman J: Origin of eye and eyelid movements during blinking. Mov Disord 2002; 17(suppl 2):S30-S32. 
31 Blumenthal TD, Cuthbert BN, et al: Committee report: guidelines for human startle eyeblink electromyographic studies. Psychophysiology 2005;42:1-15.

-32 Graham SJ, Langley RW, et al: Effects of haloperidol and clozapine on prepulse inhibition of the acoustic startle response and the N1/P2 auditory evoked potential in man. J Psychopharmacol 2001;15:243-250.

33 Nottage JF: Uncovering gamma in visual tasks. Brain Topogr 2010;23:58-71.

-34 Nottage JF, Morrison PD, Williams SC, Ffytche DH: A novel method for reducing the effect of tonic muscle activity on the gamma band of the scalp EEG. Brain Topogr 2013;26: 50-61.

35 Keren AS, Yuval-Greenberg S, Deouell LY: Saccadic spike potentials in gamma-band EEG: characterization, detection and suppression. Neuroimage 2010;49:2248-2263.
36 Hassler U, Barreto NT, Gruber T: Induced gamma band responses in human EEG after the control of miniature saccadic artifacts. Neuroimage 2011;57:1411-1421.

37 Collewijn H: Interocular timing differences in the horizontal components of human saccades. Vis Res 2001;41:3413-3423.

38 Thickbroom GW, Mastaglia FL: Presaccadic spike potential. Relation to eye movement direction. Electroencephalogr Clin Neurophysiol 1986;64:211-214.

39 De Vos M, Riès S, Vanderperren K, Vanrumste B, Alario FX, Van Huffel S, Burle B: Removal of muscle artifacts from EEG recordings of spoken language production. Neuroinformatics 2010;8:135-150.
0 Yilmaz G, Ungan P, et al: Interference of tonic muscle activity on the EEG: a single motor unit study. Front Hum Neurosci 2014;8:504.

-41 McMenamin BW, Shackman AJ, Maxwell JS, Greischar LL, Davidson RJ: Validation of regression-based myogenic correction techniques for scalp and source-localized EEG. Psychophysiology 2009;46:578-592.

42 Olbrich S, Jödicke J, Sander C, Himmerich H, Hegerl U: ICA-based muscle artefact correction of EEG data: what is muscle and what is brain? Comment on McMenamin et al. Neuroimage 2011;54:1-3, discussion 4-9.

43 Hipp JF, Siegel M: Dissociating neuronal gamma-band activity from cranial and ocular muscle activity in EEG. Front Hum Neurosci 2013;7:338.

-44 Evinger C, Manning KA: Pattern of extraocular muscle activation during reflex blinking. Exp Brain Res 1993;92:502-506. 